This item was submitted to Loughborough's Research Repository by the author.

Items in Figshare are protected by copyright, with all rights reserved, unless otherwise indicated.

\title{
Screening in Yukawa fluid mixtures
}

PLEASE CITE THE PUBLISHED VERSION

http://dx.doi.org/10.1103/PhysRevE.74.010402

\section{PUBLISHER}

(c) The American Physical Society

\section{VERSION}

VoR (Version of Record)

\section{PUBLISHER STATEMENT}

This work is made available according to the conditions of the Creative Commons Attribution-NonCommercialNoDerivatives 4.0 International (CC BY-NC-ND 4.0) licence. Full details of this licence are available at: https://creativecommons.org/licenses/by-nc-nd/4.0/

\section{LICENCE}

CC BY-NC-ND 4.0

\section{REPOSITORY RECORD}

Archer, Andrew J., Paul Hopkins, and Robert Evans. 2019. "Screening in Yukawa Fluid Mixtures". figshare. https://hdl.handle.net/2134/16024. 


\title{
Screening in Yukawa fluid mixtures
}

\author{
A. J. Archer, P. Hopkins, and R. Evans \\ H. H. Wills Physics Laboratory, University of Bristol, Bristol BS8 1TL, United Kingdom \\ (Received 27 April 2006; revised manuscript received 19 June 2006; published 18 July 2006)
}

\begin{abstract}
The effective pair potential between mesoscopic charged particles in a neutralizing background medium takes a Yukawa form $\exp (-\lambda r) / r$ with screening length $\lambda^{-1}$. We consider a dilute suspension of such Yukawa particles dispersed in a solvent with correlation length $\xi<\lambda^{-1}$ and show that the Yukawa interaction is screened if the pair potentials between solvent particles exhibit Yukawa decay with the same screening length $\lambda^{-1}$. However, if the solvent pair potentials are shorter ranged than the solute Yukawa potentials, then the effective potential between pairs of solute particles is unscreened, i.e., the effective potential between the solute particles is equal to the bare potential at large particle separations.
\end{abstract}

DOI: 10.1103/PhysRevE.74.010402

PACS number(s): 82.70.-y, 05.20.Jj, 61.20.Gy

It is well known that the effective potential between charged particles immersed in a neutralizing mobile medium is shorter ranged than the bare Coulomb interaction between the particles- the interaction is screened. At large interparticle separations $r$, the effective pair potential between particles $\phi^{e f f}(r) \propto \exp (-\lambda r) / r$. Screening effects due to the neutralizing medium are described by an inverse screening length $\lambda[1]$. Such Yukawa potentials are ubiquitous in charged colloidal systems arising in the classic linearized Poisson-Boltzmann or Derjaguin-Landau-Verwey-Overbeek (DLVO) theories for the effective potential between spherical charged colloids in solution [2]. Other systems in which effective Yukawa potentials arise include those between dust grains in plasma systems [3] and some proteins in solution [4]. The Yukawa potential not only arises in diverse physical problems, but is also interesting in its own right [5]. Note that the motivation for considering effective potentials in complex systems is driven by the fact that a theoretical treatment of the full mixture is often very difficult, particularly when there is a big size asymmetry between different components of the mixture. It is profitable to integrate out the degrees of freedom of one or more of the components, thereby incorporating formally their influence into an effective Hamiltonian for the remaining particles [6].

In this Rapid Communication, we consider a simple model for binary mixtures of charged particles suspended in a neutralizing medium. We examine a dilute suspension of Yukawa particles in a solvent with correlation length $\xi<\lambda^{-1}$ and we investigate the (additional) screening effect that the solvent particles have on the effective interaction between the solute Yukawa particles. Specifically, we consider a class of binary mixtures in which the pair potentials are of the form $\phi_{i j}(r)=\phi_{i j}^{s r}(r)+\epsilon_{i j} \exp (-\lambda r) / \lambda r$, where indices $i, j=1,2$ label the two different species and where $\phi_{i j}^{s r}(r)$ is a contribution to $\phi_{i j}(r)$ that is short ranged in comparison with the Yukawa term. The amplitudes $\epsilon_{i j}$ depend on the charges on the particles. For example, in modeling a binary mixture of charged spherical colloids, we would take $\phi_{i j}^{s r}(r)$ to be a hard-sphere interaction potential and $\epsilon_{i j} \propto Z_{i} Z_{j}$, where $Z_{i}$ is the (renormalized) charge on the colloid of species $i$ [2]. However, in what follows directly we will not make a particular choice for $\phi_{i j}^{s r}(r)$ or for the sign of $\epsilon_{i j}$. We denote species 1 as the solvent and species 2 as the solute. We focus on the limit of the number density of the solute particles $\rho_{2} \rightarrow 0$ and investigate the form of the effective potential $\phi_{22}^{e f f}(r)$ between the solute particles in this limit. In particular, we obtain the general result that when $\epsilon_{11}, \epsilon_{12} \neq 0$, so that there is a Yukawa tail proportional to $\exp (-\lambda r) / r$ present in $\phi_{11}(r)$ and $\phi_{12}(r), \phi_{22}^{e f f}(r)$ decays faster than $\phi_{22}(r)$ as $r \rightarrow \infty$ provided the solvent bulk correlation length $\xi<\lambda^{-1}$. On the other hand, when $\epsilon_{11}=\epsilon_{12}=0$ and there is no Yukawa tail in $\phi_{11}(r)$ and $\phi_{12}(r)$, the effective potential $\phi_{22}^{e f f}(r) \rightarrow \phi_{22}(r)$ for $r \rightarrow \infty$. This Yukawa "screening" effect is independent of the sign of the "charges," i.e., independent of the signs of $\epsilon_{i j}$. The results can be restated in terms of the solvent-mediated potential $W_{22}(r)$, defined via $\phi_{22}^{e f f}(r) \equiv \phi_{22}(r)+W_{22}(r)[6] . W_{22}(r)$ depends on the nature of the solvent and on the solvent-solute interaction. If $W_{22}(r) \rightarrow 0$ faster than $\phi_{22}(r)$, as $r \rightarrow \infty$, then we describe the solute-solute interaction as "unscreened". However, if $W_{22}(r)$ partially or completely cancels the bare potential $\phi_{22}(r)$ at large $r$, we describe the solute-solute interaction as "screened". In certain mixtures where the bare interactions are purely repulsive we find "superscreening", i.e., $\phi_{22}^{e f f}(r)$ can be attractive.

We may determine the effective interaction between two solute particles at infinite dilution from the solute-solute radial distribution function $g_{22}(r)$ using the well-known result [6]

$$
\beta \phi_{22}^{e f f}(r)=-\ln \left[g_{22}(r)\right],
$$

where $\beta=\left(k_{B} T\right)^{-1}$ is the inverse temperature and $g_{22}(r)$ is evaluated in the limit $\rho_{2} \rightarrow 0$. For large $r$, when $\beta \phi_{22}^{\text {eff }}(r)$ is small, it follows that

$$
\beta \phi_{22}^{e f f}(r) \sim-h_{22}(r), \quad r \rightarrow \infty
$$

where $h_{i j}(r) \equiv g_{i j}(r)-1$, and in order to determine the asymptotic behavior of $\phi_{22}^{e f f}(r)$ we must ascertain that of $h_{22}(r)$. The total pair correlation functions $h_{i j}(r)$ are related via the Ornstein-Zernike (OZ) integral equations [1] to a set of pair direct correlation functions $c_{i j}(r)$. The $\mathrm{OZ}$ equations can be solved formally in Fourier space and the solution written (for arbitrary concentration) as 


$$
\hat{h}_{i j}(q)=N_{i j}(q) / D(q),
$$

where $\hat{h}_{i j}(q)$ denotes the three-dimensional Fourier transform of $h_{i j}(r)$. The three functions share the same denominator

$$
D(q)=\left[1-\rho_{1} \hat{c}_{11}(q)\right]\left[1-\rho_{2} \hat{c}_{22}(q)\right]-\rho_{1} \rho_{2} \hat{c}_{12}(q)^{2},
$$

where $\rho_{i}$ is the density of species $i$. The numerators $N_{i j}(q)$ may be obtained from Ref. [1] or [7]. Taking the inverse Fourier transform of Eq. (3), evaluating the integral by a contour integration around a semicircle in the upper half of the complex $q$ plane, and assuming that the singularities of $\hat{h}_{i j}(q)$ are simple poles, one can express $h_{i j}(r)$ as a sum of contributions from the set of poles at $\left\{q_{n}\right\}$ in the upper half of the complex plane [7]:

$$
r h_{i j}(r)=\sum_{n} A_{i j}^{n} \exp \left(\mathrm{i} q_{n} r\right),
$$

where $A_{i j}^{n}$ is the amplitude associated with the pole at $q_{n}$. The poles are obtained from the set of solutions of $D\left(q_{n}\right)=0$. The amplitude $A_{i j}^{n}$ is related to the residue $R_{i j}^{n}$ of $q N_{i j}(q) / D(q)$ by $A_{i j}^{n}=R_{i j}^{n} / 2 \pi$. The poles are either purely imaginary, $q=i \alpha_{0}$, or occur as a conjugate pair $q= \pm \alpha_{1}+i \widetilde{\alpha}_{0}$ [7]. A purely imaginary pole gives a monotonic contribution to $r h_{i j}(r)$ of the form $A_{i j} \exp \left(-\alpha_{0} r\right)$. A conjugate pair of poles gives a damped oscillatory contribution of the form $2 \widetilde{A}_{i j} \exp \left(-\widetilde{\alpha}_{0} r\right) \cos \left(\alpha_{1} r-\tilde{\theta}_{i j}\right)$, where $\widetilde{A}_{i j}$ and $\tilde{\theta}_{i j}$ denote the amplitude and phase, respectively [7]. In general, there are an infinite number of poles. However, the asymptotic decay $r \rightarrow \infty$ is determined by the pole(s) with the smallest imaginary part $\alpha_{0}$ (or $\left.\widetilde{\alpha}_{0}\right)$.

Away from any critical points, the direct pair correlation functions $c_{i j}(r)$ are known to decay as $c_{i j}(r) \sim-\beta \phi_{i j}(r)$, $r \rightarrow \infty$ [1]. When $\phi_{i j}(r)$ has a Yukawa contribution it is convenient to separate $c_{i j}(r)$ in the following way: $c_{i j}(r)$ $=c_{i j}^{s r}(r)-\beta \epsilon_{i j} \exp (-\lambda r) / \lambda r$, which defines $c_{i j}^{s r}(r)$, the shortranged piece in $c_{i j}(r)$, dependent on the form of $\phi_{i j}^{s r}(r)$ and on the state point. In Fourier space it follows that

$$
\hat{c}_{i j}(q)=\hat{c}_{i j}^{s r}(q)-\alpha_{i j} /\left(q^{2}+\lambda^{2}\right),
$$

where $\alpha_{i j}=4 \pi \beta \epsilon_{i j} / \lambda$.

We seek the pole(s) in $\hat{h}_{i j}(q)$ with the smallest imaginary part. First, we consider the case when there is no Yukawa contribution to the solvent potentials, i.e., $\alpha_{11}=\alpha_{12}=0$. The asymptotic decay of $h_{11}(r)$ in the pure solvent of species 1 is determined by the pole(s) in $\hat{h}_{11}(q)$, given by the solution of $D_{1}(q)=1-\rho_{1} \hat{c}_{11}^{s r}(q)=0$, with the smallest imaginary part $\alpha_{0}$ and the bulk solvent correlation length $\xi=\alpha_{0}^{-1}$ [7]. Consider now the decay of $h_{i j}(r)$ in the full mixture. Making the separation of $\hat{c}_{i j}(q)$ given by Eq. (6) and substituting into Eq. (4) we obtain

$$
D(q)=A(q)+\rho_{2} \alpha_{22} D_{1}(q) / p,
$$

where $\quad p=q^{2}+\lambda^{2} \quad$ and $\quad A(q)=D_{1}(q)\left[1-\rho_{2} \hat{c}_{22}^{s r}(q)\right]$ $-\rho_{1} \rho_{2}\left[\hat{c}_{12}^{s r}(q)\right]^{2}$. The equation $D(q)=0$ has a solution $q=i\left[\lambda^{2}+\rho_{2} \alpha_{22} D_{1}(q) / A(q)\right]^{1 / 2}$, which implies that in the limit $\rho_{2} \rightarrow 0$ there is a pure imaginary pole at $q=q_{1} \equiv i \lambda$, provided the ratio $D_{1}(q) / A(q)$ remains finite in this limit. Since we have assumed that there is no Yukawa contribution in $\phi_{11}(r)$, there is no pole at $q_{1}$ for the pure solvent and $D_{1}\left(q_{1}\right)$ is nonzero and finite. If in addition, we assume that both $\hat{c}_{12}^{s r}\left(q_{1}\right)$ and $\hat{c}_{22}^{s r}\left(q_{1}\right)$ are finite it follows that in the limit $\rho_{2} \rightarrow 0$, $D_{1}(q) / A(q) \rightarrow 1$ for $q \simeq q_{1}$. The amplitude of the contribution to $h_{i j}(r)$ from this purely imaginary pole at $q_{1}$ is given by [7]

$$
A_{i j}=\frac{q_{1} N_{i j}\left(q_{1}\right)}{2 \pi D^{\prime}\left(q_{1}\right)},
$$

where the prime denotes the derivative with respect to $q$. It is straightforward to show that in the limit $\rho_{2} \rightarrow 0$, $D^{\prime}\left(q_{1}\right) \simeq-2 q_{1} D_{1}\left(q_{1}\right) / \rho_{2} \alpha_{22}$. Using this result and evaluating the numerators $N_{i j}\left(q_{1}\right)$, we find that the amplitudes of the contributions to $h_{i j}(r)$ from the purely imaginary pole at $q_{1} \equiv i \lambda$ are

$$
\begin{gathered}
A_{11}=-\frac{\hat{c}_{12}^{2}\left(q_{1}\right) \alpha_{22}}{4 \pi D_{1}^{2}\left(q_{1}\right)} \rho_{2}^{2}+O\left(\rho_{2}^{3}\right), \\
A_{12}=\frac{\hat{c}_{12}\left(q_{1}\right) \alpha_{22}}{4 \pi D_{1}\left(q_{1}\right)} \rho_{2}+O\left(\rho_{2}^{2}\right), \\
A_{22}=-\frac{\alpha_{22}}{4 \pi}+O\left(\rho_{2}\right) .
\end{gathered}
$$

These results obey the rule $A_{12}^{2}=A_{11} A_{22}$, which general considerations demand [7]. Note that in the limit $\rho_{2} \rightarrow 0$ the amplitudes $A_{11}, A_{12} \rightarrow 0$. Thus the contributions from the pole at $i \lambda$ to the decay of $h_{11}(r)$ and $h_{12}(r)$ are vanishingly small, as one would expect on physical grounds. Note further that in the same limit, $A_{22}$ tends to a nonzero constant value $-\alpha_{22} / 4 \pi=-\beta \epsilon_{22} / \lambda$ which is independent of any properties of the solvent, i.e., the pole at $q_{1} \equiv i \lambda$ gives a contribution to $r h_{22}(r)$ of the form $-\left(\beta \epsilon_{22} / \lambda\right) \exp (-\lambda r)$. Thus from Eq. (2) one finds that as $r \rightarrow \infty, \phi_{22}^{e f f}(r) \rightarrow \phi_{22}(r)$. In summary, when $\alpha_{11}=\alpha_{12}=0$, the effective interaction between the solute particles is identical to the bare interaction as $r \rightarrow \infty$; the solutesolute interaction is unscreened. Of course the argument we have presented supposes that the pole $q_{1} \equiv i \lambda$ is the leadingorder one, i.e., it has the smallest imaginary part. If the pure solvent has a correlation length $\xi>\lambda^{-1}$ then one expects the asymptotic decay of all three correlation functions $r h_{i j}(r) \sim \exp (-r / \xi)$ and $\phi_{22}^{e f f}(r)$ to be longer ranged than $\phi_{22}(r)$. Henceforward we restrict consideration to cases where $\xi<\lambda^{-1}$.

We turn now to the more realistic case when $\alpha_{11}, \alpha_{12}$ $\neq 0$, i.e., all three pair potentials have Yukawa tails. Proceeding in a similar manner as above (see also the Appendix in Ref. [8]) the denominator function (4) takes the form $D(q)=a+b / p+c / p^{2}, \quad$ where $a=\left[1-\rho_{1} c_{11}^{s r}(q)\right]\left[1-\rho_{2} c_{22}^{s r}(q)\right]$ $-\rho_{1} \rho_{2}\left[c_{12}^{s r}(q)\right]^{2}, \quad b=\left[1-\rho_{1} c_{11}^{s r}(q)\right] \rho_{2} \alpha_{22}+\left[1-\rho_{2} c_{22}^{s r}(q)\right] \rho_{1} \alpha_{11}$ $+2 \rho_{1} \rho_{2} c_{12}^{s r}(q) \alpha_{12}$, and $c=\rho_{1} \rho_{2}\left(\alpha_{11} \alpha_{22}-\alpha_{12}^{2}\right)$. One set of solutions to the equation $D(q)=0$ is given by $p_{ \pm}=-\left(b \pm \sqrt{b^{2}-4 a c}\right) / 2 a$. This leads to purely imaginary poles at $q_{ \pm}=i \alpha_{0}^{ \pm}=i \sqrt{\lambda^{2}-p_{ \pm}}$, provided we assume that the functions $c_{i j}^{s r}(q)$ are well behaved (finite and differentiable) on the 
imaginary axis around $q_{ \pm}$. The leading-order pole corresponds to $p_{-}$, and in the limit of vanishing density $\rho_{2}=0$ (i.e., $c=0)$ there is a pole at $q=i \lambda$, so that $r h_{i j}(r) \sim A_{i j}^{-} \exp (-\lambda r)$, $r \rightarrow \infty$. For small concentrations of species 2 we Taylor expand $p_{-}$in powers of $c$ and find that the leading-order pole is given by

$$
\alpha_{0}^{-}=\lambda+\frac{\alpha_{12}^{2}-\alpha_{11} \alpha_{22}}{2 \lambda \alpha_{11}} \rho_{2}+O\left(\rho_{2}^{2}\right)
$$

Using Eq. (8) we calculate the amplitudes $A_{i j}^{-}$of the contributions from this pole to the correlation functions $h_{i j}(r)$ :

$$
\begin{gathered}
A_{11}^{-}=\frac{\alpha_{12}^{2}\left(\alpha_{12}^{2}-\alpha_{11} \alpha_{22}\right)}{4 \pi \alpha_{11}^{3}}\left(\frac{\rho_{2}}{\rho_{1}}\right)^{2}+O\left(\rho_{2}^{3}\right), \\
A_{12}^{-}=-\frac{\alpha_{12}\left(\alpha_{12}^{2}-\alpha_{11} \alpha_{22}\right)}{4 \pi \alpha_{11}^{2}}\left(\frac{\rho_{2}}{\rho_{1}}\right)+O\left(\rho_{2}^{2}\right), \\
A_{22}^{-}=\frac{\left(\alpha_{12}^{2}-\alpha_{11} \alpha_{22}\right)}{4 \pi \alpha_{11}}+O\left(\rho_{2}\right) .
\end{gathered}
$$

Note that the coefficients of the leading-order terms are independent of $c_{i j}^{s r}(r)$ and that the amplitudes obey the rule [7] $\left(A_{12}^{-}\right)^{2}=A_{11}^{-} A_{22}^{-}$. In the simplest model of a charged system one expects $\alpha_{12}^{2}=\alpha_{11} \alpha_{22}$ since $\alpha_{i j} \propto Z_{i} Z_{j}$, the product of the charges on each species. We refer to this situation as the "ideal" mixing rule [8]. Then the coefficients of the leadingorder terms in Eq. (11) vanish identically and for all three $h_{i j}(r)$ the amplitudes corresponding to the pole at $q_{1}=i \lambda$ will be zero in the limit $\rho_{2} \rightarrow 0$. Thus the asymptotic decay of $h_{22}(r)$ [and therefore of $\phi_{22}^{\text {eff }}(r)$ ] is determined by the next order pole, which generally has $\alpha_{0}^{-1}=\xi<\lambda^{-1}$, so that $r \phi_{22}^{\text {eff }}(r)$ decays as $\exp (-r / \xi)$, i.e., faster than $\phi_{22}(r)$, so that the interaction between species 2 solute particles is screened. In the physical systems where $\alpha_{12}^{2} \neq \alpha_{11} \alpha_{22}$, there will still be partial screening since one expects the difference $\left(\alpha_{12}^{2}-\alpha_{11} \alpha_{22}\right)$ to be small and then $\phi_{22}^{\text {eff }}(r)$ will decay, as $r \rightarrow \infty$, with the same exponential decay length $\lambda^{-1}$ as the bare potential, but with a reduced amplitude proportional to $\left(\alpha_{12}^{2}-\alpha_{11} \alpha_{22}\right)$. We now display results for a number of model systems that confirm these general predictions.

The first class of systems is a mixture in which the particles interact via purely repulsive (point) Yukawa pair potentials for which $\phi_{i j}^{s r}(r) \equiv 0$ and $\phi_{i j}(r)=\epsilon_{i j} \exp (-\lambda r) / \lambda r$ $[8,9]$. We denote this system A. The structure and phase behavior is described in Ref. [8]. In this system the effective potential $\phi_{22}^{e f f}(r)$ decays faster than the bare potential $\phi_{22}(r)$ reflecting the Yukawa decay of the solvent (species 1) potentials (see Fig. 1). We compare with system B in which $\phi_{22}(r)$ is the same as in system A but where the solvent potentials are modified slightly to hasten the Yukawa decay at large $r$, i.e., $\phi_{11}(r)=\epsilon_{11} \exp \left(-\lambda r-\gamma(\lambda r)^{10}\right) / \lambda r$ and $\phi_{12}(r)=\epsilon_{12} \exp \left(-\lambda r-\gamma(\lambda r)^{10}\right) / \lambda r$. In both systems we choose pair potential parameters $\beta \epsilon_{11}=1, \beta \epsilon_{22}=4$ and the mixing rule $\epsilon_{12}=(1+\delta) \sqrt{\epsilon_{11} \epsilon_{22}}$ where the parameter $\delta$ measures the degree of nonideality [8]. The state point has solvent density $\rho_{1} \lambda^{-3}=3$ and solute density $\rho_{2} \lambda^{-3}=10^{-6}$,

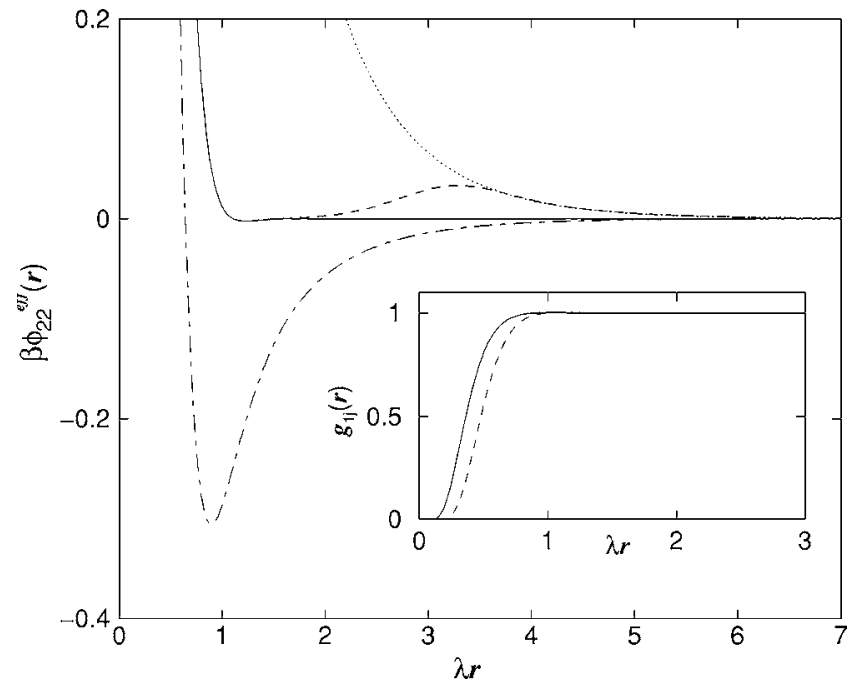

FIG. 1. The effective solute-solute potential $\phi_{22}^{e f f}(r)$ for solvent density $\rho_{1} \lambda^{-3}=3$ and $\rho_{2} \rightarrow 0$ calculated using the HNC closure. Solid line: system A, the point Yukawa mixture, with $\delta=0$ and dot-dashed line with nonideality parameter $\delta=0.1$. The dashed line is for system $\mathrm{B}(\delta=0)$ where the solvent potentials decay faster than for the Yukawa case. Results are compared to the bare potential $\phi_{22}(r)$ (dotted line). The inset displays the radial distribution functions $g_{11}(r)$ (solid line) and $g_{12}(r)$ (dashed line) for $\delta=0$. On this scale, there is no visible difference between results in systems A and B.

corresponding to the dilute limit. In system B we choose $\gamma=10^{-5}$, which is sufficiently small that the solvent radial distribution functions $g_{11}(r)$ and $g_{12}(r)$ are almost indistinguishable from those in system A [10]. Figure 1 displays $\phi_{22}^{\text {eff }}(r)$ calculated using the hypernetted chain (HNC) closure to the $\mathrm{OZ}$ equations [1], which is expected to be a very reliable approximation for this model fluid [8]. For system A with $\delta=0$ (solid line) there is screening; $\phi_{22}^{\text {eff }}(r)$ is much shorter ranged than the bare potential $\phi_{22}(r)$ (dotted line). In system B with $\delta=0$ (dashed line) $\phi_{22}^{e f f}(r)$ is indistinguishable from that in system A at small $r$, but for $\lambda r \geqslant 2$ the results differ significantly - there is a maximum near $\lambda r=3.3$ and for $\lambda r \gtrsim 4, \phi_{22}^{e f f}(r) \rightarrow \phi_{22}(r)$; the solute-solute interaction is unscreened. For $\delta=0.1$ in system A (dot-dashed line) $\phi_{22}^{e f f}(r)$ has a pronounced minimum near $\lambda r=0.9$ and is attractive for larger $r$ as the amplitude $A_{22}^{-}>0$ - see Eq. (11). We refer to this scenario as superscreening. It is remarkable that such an effective attraction arises in a system where all the bare interactions are purely repulsive and this constitutes a dramatic signal that for $\delta>0$ the fluid exhibits liquid-liquid phase separation when the densities of the two components are sufficiently high [8].

The second class of systems is that in which the particles have a hard-core interaction mimicking (charged) spherical colloids. In system $\mathrm{C}$ the pair potentials are of the form $\phi_{i j}(r)=\phi_{H S}(r)+\epsilon_{i j} \exp (-\lambda r) / \lambda r$, where $\phi_{H S}(r)=\infty$ for $r<\sigma$ and 0 for $r>\sigma$, the hard-sphere diameter [11]. We choose the parameters $\lambda=0.3 \sigma^{-1}, \beta \epsilon_{11}=0.1, \beta \epsilon_{12}=0.2, \beta \epsilon_{22}=0.4$, corresponding to the case where the sign of the charge is the same on both species but the magnitude $Z_{2}=2 Z_{1}$. We compare with system $\mathrm{D}$ in which the charges on the particles have the same 


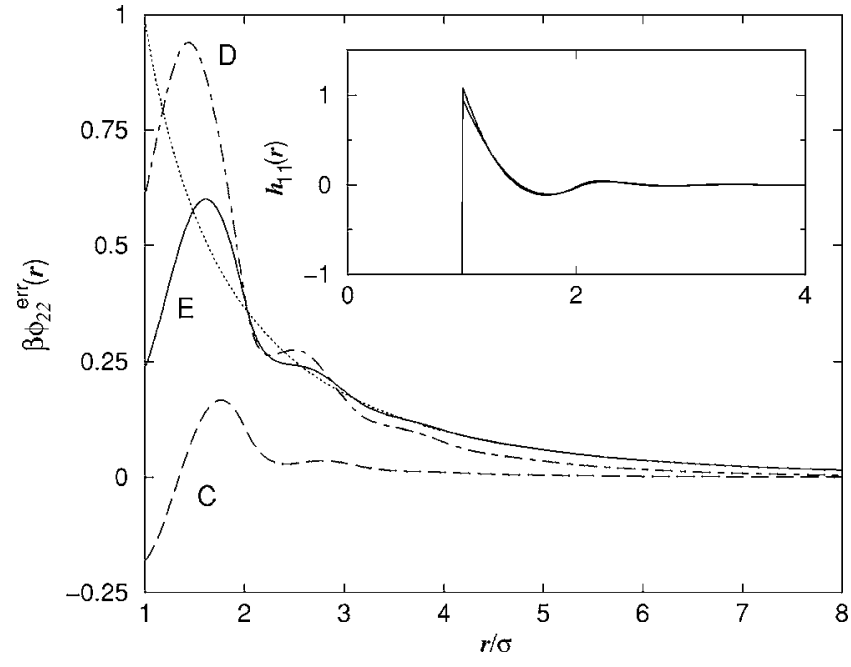

FIG. 2. The effective potential $\phi_{22}^{e f f}(r)$ for solvent density $\rho_{1} \sigma^{3}=0.5$ and $\rho_{2} \rightarrow 0$ calculated using the PY closure for hard-core Yukawa mixtures. Dashed line, system $\mathrm{C}\left(\beta \epsilon_{11}=0.1, \beta \epsilon_{22}=0.4\right.$, $\left.\beta \epsilon_{12}=0.2\right)$; dot-dashed line, system $\mathrm{D}\left(\beta \epsilon_{12}=-0.2\right)$; and solid line, system $\mathrm{E}\left(\beta \epsilon_{11}=0, \beta \epsilon_{12}=0, \beta \epsilon_{22}=0.4\right)$. In systems $\mathrm{C}$ and $\mathrm{D}, \phi_{22}^{\text {eff }}(r)$ is shorter ranged than the bare potential $\phi_{22}(r)$ (dotted line) but in system $\mathrm{E}, \phi_{22}^{\text {eff }}(r) \simeq \phi_{22}(r)$ for $r \geqslant 4 \sigma$; there is no screening. The inset displays the corresponding total correlation function $h_{11}(r)$ for systems C, D, and E.

magnitude as in system $C$ but the opposite sign, $\beta \epsilon_{12}=-0.2$; all the other parameters remain the same. The final comparison is with system $\mathrm{E}$ in which the solvent (species 1) particles are neutral hard spheres: $\epsilon_{11}=\epsilon_{12}=0$ while $\phi_{22}(r)$ is the same as in systems $\mathrm{C}$ and $\mathrm{D}$ (with $\beta \epsilon_{22}=0.4$ ). In Fig. 2 we display the results for $\phi_{22}^{e f f}(r)$ calculated using the PercusYevick (PY) closure to the OZ equations [1] for these three systems at the solvent density $\rho_{1} \sigma^{3}=0.5$ and solute density $\rho_{2} \sigma^{3}=10^{-6}$. The PY closure should be reasonably accurate for such hard-core systems with additional weak Yukawa tails. We see that in system $\mathrm{C}$ (dashed line) and D (dotdashed line) $\phi_{22}^{\text {eff }}(r)$ decays much more rapidly than in system $\mathrm{E}$ (solid line) where $\phi_{22}^{e f f}(r) \simeq \phi_{22}(r)$ for $r \gtrsim 4 \sigma$, i.e., the solute-solute interaction is unscreened.

Screening occurs in both systems C and D. Perhaps surprisingly, the screening effect is stronger in system $\mathrm{C}$ where the solvent particles have the same sign charge as the solute particles. Note that in case D where the solute particles have the opposite sign charge to the solvent particles, $\phi_{22}^{e f f}(r)$ can become quite large and positive at small and intermediate values of $r$, as the magnitudes of the charges are increased. In this case the screening effect discussed here, which applies to the ultimate $r \rightarrow \infty$ decay of $\phi_{22}^{e f f}(r)$, becomes visible only at very large values of $r$.

In this Rapid Communication we have shown that in Yukawa mixtures the interaction between particles of one species can be screened by the other species. The screening effect does not depend on the sign of the charges on the particles_-see Eq. (11)—one observes like-charge screening, in contrast with standard Coulombic screening. Our results may have implications for the effective interactions in models of charged binary colloidal suspensions.

A.J.A. acknowledges the support of EPSRC under Grant No. GR/S28631/01.
[1] J.-P. Hansen and I. R. McDonald, Theory of Simple Liquids, 3rd ed. (Academic, London, 2006).

[2] See, e.g., J.-P. Hansen and H. Löwen, Annu. Rev. Phys. Chem. 51, 209 (2000), and references therein.

[3] A. Piel and A. Melzer, Adv. Space Res. 29, 1255 (2002), and references therein.

[4] See, e.g., C. F. Wu and S. H. Chen, J. Chem. Phys. 87, 6199 (1987).

[5] J. S. Rowlinson, Physica A 156, 15 (1989).

[6] See, e.g., C. N. Likos, Phys. Rep. 348, 267 (2001).

[7] R. Evans et al., J. Chem. Phys. 100, 591 (1994).
[8] P. Hopkins et al., J. Chem. Phys. 124, 054503 (2006).

[9] For a discussion of the structure of a one-component point Yukawa fluid see, e.g., P. Hopkins et al., Phys. Rev. E 71, 027401 (2005), and references therein.

[10] Note that for this state point the decay of $g_{11}(r)$ in the pure solvent is damped oscillatory with a small amplitude—see Fig. 4 in Ref. [8].

[11] For a discussion of the structure and phase behavior of a onecomponent hard-core Yukawa fluid, see, e.g., A. P. Hynninen and M. Dijkstra, Phys. Rev. E 68, 021407 (2003), and references therein. 